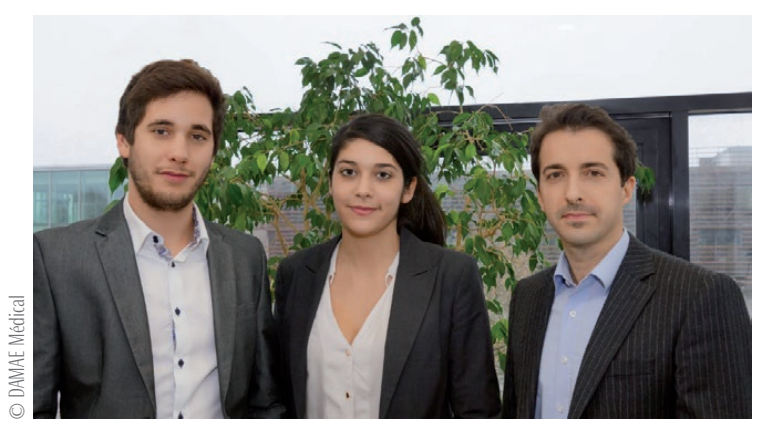

\title{
DAMAE Medical : diagnostiquer les tumeurs cutanées sans biopsie
}

De gauche à droite : David Siret, Anaïs Barut et Arnaud Dubois

Le prototype de diagnostic rapide et non invasif des cancers de la peau fondé sur l'imagerie optique proposé par DAMAE Medical est prêt. La start-up vise une commercialisation en 2016.

Le diagnostic du cancer de la peau nécessite un prélèvement de tissu, analysé ensuite au microscope sous la forme de coupes histologiques. C'est la biopsie. Et si l'analyse pouvait être réalisée directement sur le patient, avec la même exigence de précision ? C'est ce que propose désormais la start-up issue du CNRS, DAMAE Medical, dont le dispositif d'imagerie optique à haute résolution fera l'objet d'essais cliniques avant la fin de l'année.

Plus précisément, celui-ci est fondé sur la tomographie par cohérence optique (OCT) [1] : une image est reconstruite par interférence de deux faisceaux de lumière, dont l'un est réfléchi par le tissu à analyser. Cette technique est notamment utilisée avec succès en ophtalmologie, où elle permet d'obtenir des images de la rétine.

Mais, contrairement à l'œil, la peau est un milieu diffusant, semblable à du verre dépoli : les rayons de lumière ne s'y propagent pas en ligne droite et perdent rapidement toute "mémoire » de leur lieu d'origine. D'où a priori des images totalement floues. Alors même que, si une résolution à l'échelle des couches de la rétine est suffisante en ophtalmologie, l'examen d'une tumeur de la peau s'effectue à l'échelle cellulaire. "Un milieu diffusant, la nécessité de résoudre à l'échelle du micromètre sur un millimètre d'épaisseur, et le tout en temps réel, nous cumulons trois difficultés", résume Arnaud Dubois, enseignant-chercheur au Laboratoire Charles Fabry (CNRS, IOGS) et cofondateur de DAMAE Medical.

Il n'en dira pas plus sur ce qui a permis concrètement de surmonter ces difficultés. Un brevet est en effet actuellement en

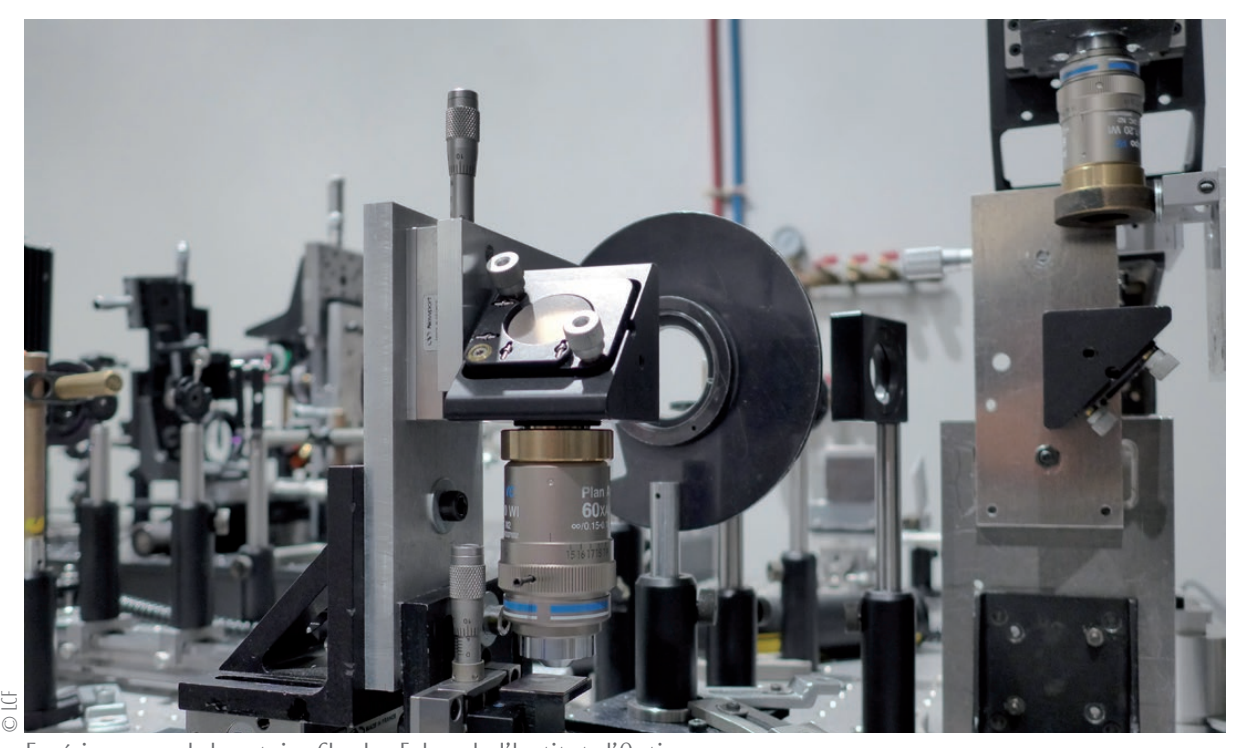

Expériences au Laboratoire Charles Fabry de l'Institut d'0ptique.

cours de dépôt. Cette capacité à reconstituer une image à partir des seuls photons non diffusés (environ un sur un milliard envoyés !), est l'aboutissement de plus de dix ans de recherche fondamentale.

Une chose est sûre, un prototype fonctionne désormais au laboratoire. Il sera prochainement mis en service à l'Hôpital Saint-Louis, à Paris, et au CHU de Saint-Étienne, lors d'essais cliniques. S'en suivra une étape de miniaturisation, afin que le dispositif se présente sous la forme d'une sonde portable à appliquer directement sur la peau. "L'objectif n'est pas d'éliminer complétement les biopsies, explique le physicien, mais de proposer un outil en amont qui permettra de limiter cette méthode invasive aux cas potentiellement problématiques qui nécessitent un examen plus approfondi."

Pour que cette petite révolution annoncée en dermatologie devienne une réalité, la structure est prête. En plus de ses trois fondateurs - David Siret, Anais Barut et Arnaud Dubois, les deux derniers étant de jeunes diplômés de la "Filière Innovation Entrepreneurs " de l'Institut d'Optique Graduate School - DAMAE Medical emploie trois autres personnes à temps complet : deux ingénieurs, dont un ingénieur de valorisation du CNRS, et une attachée clinique qui fait le lien avec le monde médical. Pour preuve du sérieux de l'entreprise, DAMAE a déjà été récompensée par de nombreux prix : à l'été 2014, elle a fait partie des lauréats du Concours mondial d'innovation ; puis, en juin dernier, elle a remporté le prix EDF Pulse dans la catégorie "Santé ". La Banque publique d'investissement lui a par ailleurs alloué récemment une subvention d'aide au lancement de 250000 euros. Et, en tant que lauréate 2014 du prix international de la fondation Altran pour l'innovation, elle reçoit l'aide d'ingénieurs pour l'industrialisation et le montage.

De quoi faire bientôt du dépistage du cancer de la peau un geste médical presque anodin!

Mathieu Grousson, journaliste [1] C. Boccara, Reflets de la physique 13 (2009) 10-13.

\section{- Contact}

\section{Arnaud Dubois}

(arnaud.dubois@institutoptique.fr) 